\title{
An expanded seed sequence definition accounts for full regulation of the hid 3' UTR by bantam miRNA
}

\author{
ALI NAHVI, CHRISTOPHER J. SHOEMAKER, and RACHEL GREEN \\ Department of Molecular Biology and Genetics, Howard Hughes Medical Institute, Johns Hopkins University School of Medicine, Baltimore, \\ Maryland 21205, USA
}

\begin{abstract}
MicroRNAs (miRNAs) are an abundant class of $\sim 22$ nucleotide (nt) long noncoding RNAs that negatively regulate gene expression post-transcriptionally through imperfect base-pairing interactions with sequences in the target messenger RNA (mRNA). We examined the interactions of the bantam miRNA with the 3' untranslated region (UTR) of the hid mRNA, and a synthetic derivative, in Drosophila S2 cells in order to define the relative contributions of proposed bantam binding sites. The contribution of the bantam miRNA to repression of reporter constructs carrying different 3' UTRs was evaluated by measuring derepression of reporter expression following the transfection of bantam complementary oligoribonucleotides (anti-bantam). Systematic excision of bantam miRNA target sequences in the hid 3' UTR identified by commonly used miRNA target prediction programs failed to relieve repression to the extent predicted by the anti-bantam experiment. However, removal of additional bantam complementary sequences (with a "seed" match to nucleotide 3-9) derepressed the reporter constructs to the full extent, arguing for a less narrow definition of the seed sequence. Further support for the potential contribution of the 3-9 seed register to microRNA-mediated gene regulation is provided by the experimental validation of several novel bantam targets identified with a more relaxed search algorithm.
\end{abstract}

Keywords: 3' UTR; Drosophila melanogaster; gene regulation; miRNA

\section{INTRODUCTION}

MicroRNAs (miRNAs) are an evolutionarily conserved class of short ( $\sim 22$ nucleotides [nt]) RNAs known to play critical roles in a variety of cellular processes including differentiation, apoptosis, and development (Esquela-Kerscher and Slack 2006; Bushati and Cohen 2007; Kato and Slack 2008). miRNAs, through their association with Argonaute (Aravin et al. 2003) proteins in the RNA-induced silencing complex (RISC), base pair with sites of partial complementarity located in the 3' UTR of target mRNAs to down-regulate gene expression (Jackson and Standart 2007; Nilsen 2007; Pillai et al. 2007). Recent studies have also argued that miRNAs can positively regulate gene expression under certain stages of the cell cycle (Vasudevan et al. 2007). The molecular mechanism through which the RISC complex mediates such posttranscriptional gene regulation (negative or positive) has been the subject of considerable study and controversy. Indeed,

Reprint requests to: Rachel Green, Department of Molecular Biology and Genetics, Howard Hughes Medical Institute, Johns Hopkins University School of Medicine, 725 North Wolfe Street, Baltimore, MD 21205, USA; e-mail: ragreen@jhmi.edu; fax: (410) 955-9124.

Article published online ahead of print. Article and publication date are at http://www.rnajournal.org/cgi/doi/10.1261/rna.1565109. almost each new study reports a different root cause for the observed repression of gene expression including translation initiation, elongation, deadenylation, and cellular localization (Olsen and Ambros 1999; Bagga et al. 2005; Pillai et al. 2005; Behm-Ansmant et al. 2006a,b; Giraldez et al. 2006; Nottrott et al. 2006; Petersen et al. 2006; Wu et al. 2006; Chendrimada et al. 2007; Kiriakidou et al. 2007; Mathonnet et al. 2007; Thermann and Hentze 2007; Eulalio et al. 2008). These studies have generally depended on reporter systems that exhibit miRNA-mediated repression effects of up to 10 -fold in cultured cells or in cell free extract. While these effects are reproducible, their magnitude is small enough to make biochemical characterization a challenge. Moreover, molecular dissection of such a complex process is difficult because of the overall lack of detailed knowledge about the rates of individual steps, thus making it a challenge to design a meaningful experiment as recently discussed (Nissan and Parker 2008). A primary goal of the studies presented here was the development of robust reporters for analysis of miRNAmediated repression for more detailed biochemical studies.

Despite these mechanistic controversies, it is abundantly clear that miRNAs play widespread and substantial roles in eukaryotic biology. To date, $>600,300$, and 150 miRNAs have been identified in the human, zebrafish, and fruitfly 
genomes, respectively, and each is thought to target multiple genes in the genome through direct interactions with binding sites located primarily within the 3' UTRs of target genes and are thought to impact their evolution (Farh et al. 2005; Stark et al. 2005). In metazoans, targets are typically thought to have overall imperfect complementarity with miRNAs, but maintain high complementarity in the region between nucleotides 2 and 8 at the $5^{\prime}$ end of the miRNA (the so-called "seed" sequence) and the target (Lewis et al. 2003, 2005; Brennecke et al. 2005). The broad features of these interactions, as initially discerned for the genetically defined interaction in Caenorhabditis elegans between the lin-4 miRNA and the lin-14 3' UTR (Lee et al. 1993; Wightman et al. 1993), are largely preserved in the few other genetically characterized miRNA pairing interactions in C. elegans and in Drosophila melanogaster (Reinhart et al. 2000; Brennecke et al. 2003). Building on these general rules derived from a handful of genetic examples and functional studies, bioinformatic approaches have taken on the larger task of identifying targets for the growing class of miRNAs. The basic premise of these approaches is to identify targets with regions complementary to the seed sequences of known miRNAs, with emphasis placed on sequences that are conserved across organisms (Lewis et al. 2003, 2005; Stark et al. 2003; Grün et al. 2005). A number of such algorithms have been developed, all of which rely heavily on seed matches (from nucleotides 2 to 8 of the miRNA), but with differential emphasis placed on other features such as complementarity with the miRNA $3^{\prime}$ end or the predicted structure of the $3^{\prime}$ UTR itself (Lewis et al. 2003; Brennecke et al. 2005; Krek et al. 2005; Griffiths-Jones et al. 2006; Kertesz et al. 2007).

These general features of target prediction guide the field in thinking about the breadth of miRNA impact on biology and significantly influence the design of experiments aimed at deciphering mechanism. However, few studies (Brennecke et al. 2005; Didiano and Hobert 2006, 2008) have systematically dissected the features of the miRNA:target interaction that are critical to the regulation of gene expression. And, in some of those cases that have been studied, the predictive power of the various algorithms appears to be poor in certain specific in vivo contexts (Didiano and Hobert 2008). As a counterbalance, two recent proteomic studies provide considerable support for target prediction algorithms and their ability to identify strong candidate genes targeted by miRNAs (Baek et al. 2008; Selbach et al. 2008). Interestingly, however, both studies concede that target conservation may not be an essential criterion for predicting in vivo responsiveness, thus opening the gates for a considerably wider selection of targets.

In our efforts to develop reporter constructs for mechanistic dissection of miRNA-mediated translational repression, we focused on the genetically identified interaction between the bantam miRNA and the proapoptotic gene head involution defective (hid). The bantam miRNA is predicted to regulate $>70$ genes in $D$. melanogaster, with the 2.25-kb-long hid 3' UTR containing anywhere from one to six predicted bantam sites, depending on the target prediction program employed-the widely used TargetScan, for example, predicts four separate bantam binding sites in the hid 3' UTR while miRanda predicts only one. Previous experiments relying on a GFP transgene reporter fused to the hid 3' UTR provided evidence that at least one of the identified sites (hid1 and/or hid4) is important for repression in the fly (Brennecke et al. 2003).

We constructed reporters carrying either a synthetic 3' UTR, composed of six tandem bantam miRNA binding sites, or the full-length endogenous target hid mRNA 3' UTR containing multiple predicted bantam target sequences. In both cases, we assessed the contribution of microRNA-mediated repression in two ways: first with complementary oligoribonucleotides targeting endogenous bantam miRNA (anti-bantam) and second by removing target sequences from the reporter constructs themselves. The behavior of the synthetic 3' UTR was consistent with expectations-application of anti-bantam relieved repression of the reporter gene to the same general extent as complete removal of the bantam target sequences. Parallel analyses with the reporter carrying the natural hid 3' UTR did not yield such consistent results-systematic removal of target sequences identified by commonly used miRNA target prediction programs failed to relieve repression to the extent predicted by the anti-miRNA experiment. Here we present evidence suggesting that the most commonly used miRNA target prediction programs may underestimate the number of potentially functional in vivo targets.

\section{RESULTS}

\section{Bantam-mediated regulation of a synthetic reporter construct harboring tandemly arrayed microRNA binding sites}

Here we use the genetically defined interaction between the bantam microRNA and the $3^{\prime}$ UTR of the proapoptotic gene hid as a starting point for the development of reporter constructs for mechanistic analysis of miRNA-mediated gene regulation (Brennecke et al. 2003). Each miRNA target prediction program easily identifies hid as a target for bantam with one to six sites predicted (miRanda and PicTar/PITA, respectively). We selected two of the hid gene bantam binding sites (hid1 and hid4) (Brennecke et al. 2003) for use in the construction of a synthetic 3' UTR that would likely respond to endogenously expressed or exogenously supplied bantam, as reported in a variety of other systems (Zeng et al. 2002; Doench et al. 2003; Pillai et al. 2005). The reporter construct includes the Renilla luciferase gene (R-Luc) fused to a relatively short 3' UTR (200 nt) comprised of six total bantam sites iteratively arrayed (hid1-hid4-hid1-hid4-hid1-hid4) (referred to as R-Lucsynthetic) (Fig. 1A). As a control, the 3' UTR of the reporter 
construct was cloned in the reverse direction, generating mRNA of the same overall length but lacking known bantam binding sites (referred to as R-Luc-flipped). The polyadenylation site for both constructs is an SV40 sequence located within the vector sequences.

The two expression constructs (synthetic and flipped) were co-transfected with a control plasmid expressing firefly luciferase (F-Luc) into D. melanogaster S2 cells, where bantam is known to be expressed at reasonably high levels (Brennecke et al. 2003). Following transfection (48 h), Renilla and firefly luciferase expression levels were measured and normalized to assess potential microRNAmediated regulation. Initial experiments indicated that the flipped R-Luc construct was generally expressed at higher levels than the synthetic construct, consistent with microRNA-mediated regulation acting through the bantam sites. Evaluation of overall RNA levels by qRT-PCR (data not shown) for the two mRNAs (R-Luc-synthetic and -flipped) revealed at most minor differences (on the order of 20\%), consistent with earlier reports (Brennecke et al. 2003; Behm-Ansmant et al. 2006a).

To identify conditions where bantam-mediated regulation might be most robust, we titrated the amount of R-Luc construct transfected over a wide range (from 0.5 to $100 \mathrm{ng}$ per well of a 24-well plate) while always transfecting the same amount (1 ng) of control F-Luc plasmid (Fig. 1B). The dose dependence of the differential response of the two constructs (synthetic versus flipped) was maximized at $\sim 30$ - to 40 -fold at low concentrations of reporter ( $2 \mathrm{ng} /$ well). These data are consistent with the idea that components important for microRNA-mediated gene regulation might be limiting (Mathonnet et al. 2007).

To directly confirm that the observed differential expression of the synthetic and flipped constructs reflects a specific microRNA-mediated effect, we examined how the synthetic and flipped reporter constructs respond to exogenously transfected antisense oligoribonucleotides (antimiRNA) that abrogate the function of specific microRNAs through direct hybridization (Meister et al. 2004; Krutzfeldt et al. 2005; Leaman et al. 2005). A panel of anti-miRNAs was chosen to target bantam, several abundant microRNAs in Drosophila (miR-1, miR-6, miR-184) (Aravin et al. 2003) and finally, as a nonspecific control, lin-4 from C. elegans, since there is no known target for this anti-miRNA in Drosophila. The anti-miRNAs that we utilized throughout this study contained 2'-O-methyl ribonucleotides and
A

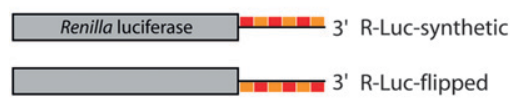

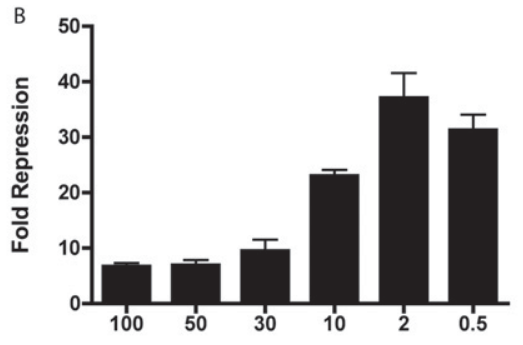

Reporter plasmid, ng

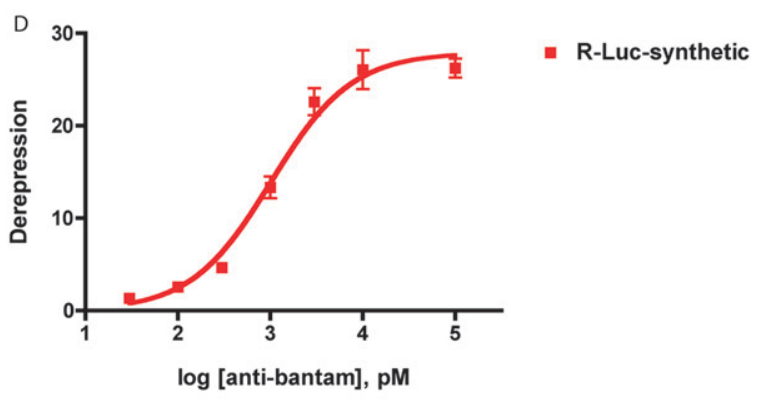

FIGURE 1. bantam miRNA-mediated translational repression of synthetic reporter mRNAs in D. melanogaster S2 cells. (A) Schematic representation of reporter constructs carrying synthetic 3' UTRs. (B) Differential expression activity of R-Luc-synthetic and R-Luc-flipped constructs is dose dependent. A range, $100 \mathrm{ng} /$ well to $0.5 \mathrm{ng} /$ well, of R-Luc reporter DNA plasmids was tested in 24-well plates while keeping the internal transfection control F-Luc plasmid constant. Normalized Renilla/firefly activity was measured for each construct with fold repression representing the ratio of R-Luc-flipped/R-Luc-synthetic. (C) Differential expression of R-Luc-synthetic and R-Luc-flipped reporters is specifically mediated by bantam miRNA. R-Luc-synthetic reporter DNA plasmid was co-transfected with $100 \mathrm{nM}$ 2'-O-methylated RNA oligonucleotides (anti-miRNA) complementary to one of several endogenous D. melanogaster miRNAs (bantam, miR-1, miR-184, miR-6) or to the C. elegans lin-4 miRNA. Renilla luciferase values (normalized to firefly values) were normalized relative to the control +anti-lin-4 sample. $(D)$ Titration of R-Lucsynthetic construct with anti-bantam RNA oligonucleotides yields maximal derepression that mirrors reporter titration in $A$. For each panel, values shown represent the mean $\pm \mathrm{SD}$ from three independent experiments. 
phosphorothioate linkages, but were not derivatized with cholesterol as in some published "antagomir" studies (Krutzfeldt et al. 2005). While expression of the flipped R-Luc construct was unaffected by our anti-miRNA panel (data not shown), the synthetic R-Luc construct was specifically derepressed by anti-bantam relative to the other anti-miRNAs (Fig. 1C). Titration of anti-bantam revealed a maximal derepression of the R-Luc construct of $\sim 30$-fold (Fig. 1D), similar to the overall difference in expression observed above when comparing the synthetic and flipped R-Luc constructs in the absence of anti-miRNA oligoribonucleotides (Fig. 1B). In addition, we demonstrated that overexpression of bantam itself (from a transfected construct expressing pre-miRNA) increased the observed level of repression by an order of magnitude when high amounts (50 ng) of reporter construct were transfected (data not shown).

As a further test of the model that regulation of the synthetic reporter constructs described above reports on miRNA-mediated repression, we asked whether the levels of repression are dependent on the miRNA specific Argonaute protein, AGO1, and not AGO2, as previously reported (Rehwinkel et al. 2006). AGO1 and AGO2 were depleted in S2 cells by bathing with dsRNA targeting the respective mRNAs. qRT-PCR confirmed that the dsRNAs were specific to the indicated genes and knocked down the mRNA levels of $A G O 1$ and $A G O 2$ by $70 \%$ and $80 \%$, respectively (data not shown). The AGO1 knockdown reduced the level of repression from 75-fold to threefold from R-Luc-synthetic 3' UTR reporters whereas with the $\mathrm{AGO} 2$ knockdown, the degree of repression remained largely unaffected $(<2$-fold difference in repression levels between AGO2 and control YFP dsRNA) (Supplemental Fig. S1).

\section{Natural 3' UTR reporter constructs are regulated by bantam in $\mathrm{S} 2$ cells}

Because of our interest in exploring miRNA-mediated gene regulation in a more natural situation, we next generated a reporter construct that carried the full-length 2.25 -kb-long 3' UTR of the hid gene including its natural polyadenylation site (Fig. 2A, R-Luc-hid; Grether et al. 1995). To ask whether this more natural construct is regulated by bantam, as predicted by genetic analysis and all target prediction programs (summarized in Supplemental Fig. S2), we transfected the reporter (R-Luc-hid) and control construct (FLuc) into S2 cells and challenged them with the panel of anti-miRNAs to confirm the specificity of the response. Consistent with the results described above for R-Lucsynthetic, the R-Luc-hid construct was specifically derepressed by anti-bantam, with a maximal derepression of around four- to sevenfold (Fig. 2B). To further explore the magnitude of regulation of more typical gene targets containing a single putative bantam binding site in their 3' UTR (as identified by TargetScan), we constructed three more reporters with natural 3' UTRs carrying their own polyadenylation sites (R-Luc-Gli, R-Luc-sca, and R-Luc$H L H m \delta$ ). As a control, we also generated a reporter construct with a natural 3' UTR not predicted to contain any bantam binding sites (R-Luc-TER94). Each of the constructs containing a single predicted bantam target site responded specifically and rather equivalently to the
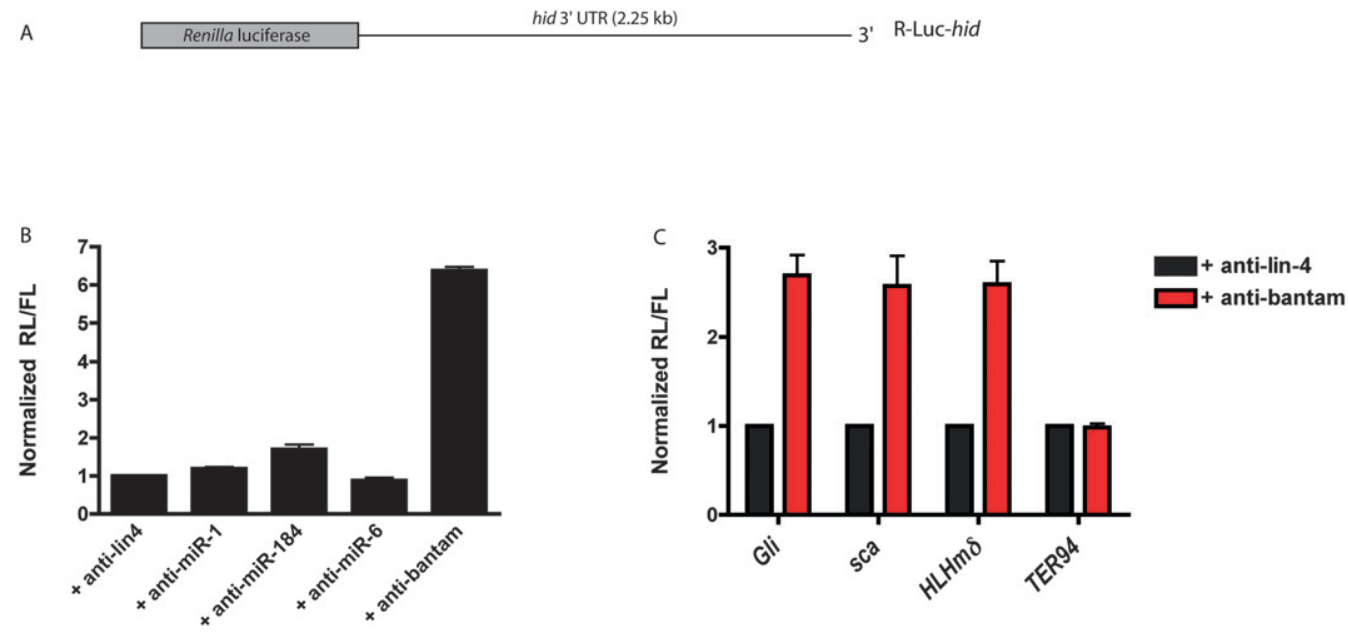

FIGURE 2. Bantam-mediated regulation of reporter mRNAs carrying full-length D. melanogaster $3^{\prime}$ UTRs. (A) Schematic of reporter construct harboring the D. melanogaster hid 3' UTR. A 2.65-kb fragment encompassing the full-length hid 3' UTR and its natural polyadenylation signal was appended to Renilla luciferase (R-Luc-hid) and (B) tested for bantam regulation by co-transfecting the reporter construct (5 ng/well) with 100 $\mathrm{nM}$ 2'-O-methylated RNA oligonucleotides (anti-miRNA). (C) Other putative bantam target genes identified by TargetScan respond to antibantam oligonucleotide. The full-length $3^{\prime}$ UTRs, including their own polyadenylation signals, of $D$. melanogaster genes Gli, sca, and $H L H m \delta$ were fused to Renilla luciferase and tested for a specific response to anti-bantam. The TER94 3' UTR, not predicted to have any bantam binding sites, was tested as a control. In both $B$ and $C$, Renilla values were normalized to firefly values and the specific response to anti-bantam was determined by dividing Renilla values in the presence of anti-bantam by the Renilla values in the presence of control anti-lin-4 $(+$ anti-lin- $4=1.0)$. 
application of anti-bantam (relative to the control, anti-lin-4) with a derepression of $\sim 2.5$ - to threefold while the control R-Luc-TER94 construct exhibited no change (Fig. 2C).

\section{Deletion of seed match sequences from target 3' UTRs provides insights into limitations of current prediction programs}

As a simple first experiment, we focused our attention on the R-Luc-HLHm $\delta$ construct. The natural HLHm $\delta 3^{\prime}$ UTR is $\sim 380 \mathrm{nt}$ in length and is predicted by TargetScan to contain a single bantam binding site. As anticipated, when the bantam binding site (the "seed" binding sequence) was deleted, the construct no longer exhibited any response to antibantam (Supplemental Fig. S3). These data are consistent with the idea that bantammediated regulation of this gene is dictated by this single computationally identified binding site. Studies by a number of groups have had similar successes in eliminating miRNA-mediated repression of particular constructs, though these experiments have rarely been performed in the background of the complete 3' UTR (Lewis et al. 2003; Doench and Sharp 2004; Wang et al. 2006).

Armed with these positive results for $H L H m \delta$, we next attempted to delete the bantam binding elements from the hid 3' UTR to generate a control construct for the R-Luchid reporter described above. As an initial attempt, the "seed" elements of the four bantam target sites identified by TargetScan were systematically deleted from the 2.25kb-long hid 3' UTR (Fig. 3A, R-Luc-hids4). A comparison of R-Luc expression levels from the wild-type and mutant R-Luc-hid constructs showed a modest 2.5-fold difference (Fig. 3B), substantially lower than the maximal level of regulation defined by the anti-bantam experiment above (Fig. 2B). As it seemed possible that deletion of seed match sequences only would be insufficient to disrupt bantam targeting to these sites, another construct was made with a larger deletion, including the seed match sequence region and some extended $3^{\prime}$ end miRNA complementary context, at each putative bantam target. As for the R-Luc-hid $\Delta 4$ construct, we observed about a twofold derepression of RLuc expression with these more extensive deletions (data not shown). These data suggested that there might be other bantam target sequences in this natural $3^{\prime}$ UTR not identified by TargetScan as other prediction programs yield a different, albeit partially overlapping, set of predicted targets in the hid 3' UTR (Fig. 3A; Supplemental Fig. S2).
To address this possibility, we scanned the hid $3^{\prime}$ UTR for other potential bantam binding sites by looking for sequence blocks complementary to nucleotides $1-7,2-8,3-9$, and 4-10 of bantam, allowing for up to a single wobble pairing interaction within the contiguous complementary region. We reasoned that complementarity beyond nucleotide 10 should not be considered, as such an interaction would likely prevent the mRNA from being targeted for classical miRNA-mediated regulation (Selbach et al. 2008). In addition to the four sites predicted by TargetScan, two additional 7-nt matches to nucleotides 2-8 of bantam, one containing a single wobble base pair (U:G), and two additional 7-nt-long matches to nucleotides 3-9 of bantam were identified in the hid 3' UTR (Supplemental Fig. S2). The latter sites are of particular interest since no current target prediction algorithm includes this class of site. We started the subsequent analysis by deleting first the two additional 2-8-nt matches to generate R-Luc-hids6 and found that this construct exhibited a still incomplete level of derepression ( $\sim 3$-fold, data not shown). However, deletion of the remaining two 3-9-nt match sequences (construct R-Luc-hids8) derepressed R-Luc expression to the full extent observed in the presence of anti-bantam (Figs. 2B, 3B). Furthermore, the R-Luc-hid $\Delta 8$ construct displays a minimal increase in luciferase expression in 
response to anti-bantam when compared to R-Luc-hid or R-Luc-hid $\Delta 4$ (Supplemental Fig. S4). The equivalence of these responses argues that all contributing bantam binding sites have been taken into account in this final deletion construct, R-Luc-hids8.

\section{Genes containing 3-9 seed matches not identified by target prediction programs respond to bantam}

Deletion analysis of the hid 3' UTR suggested that sequences complementary to nucleotides 3-9 of bantam might act as competent miRNA targeting sites. To test this hypothesis directly, we generated a reporter construct consisting of Renilla luciferase fused to a short 3' UTR that includes five copies of the putative bantam binding sites from the hid $3^{\prime}$ UTR with complementary pairing to nucleotides 3-9 of bantam herein referred to as R-Luc-synthetic(3-9). We compared this construct to an equivalent version with the original array of 2-8 target sites [R-Luc-synthetic(2-8)] and saw that both were repressed to similar levels in S2 cells (Fig. $4 \mathrm{~A})$. These data indicate that a 3' UTR with complementarity to nucleotides 3-9 of bantam also confers repression.

To test whether sequences complementary to bantam nucleotides 3-9 are functional in their native context, we searched for genes that have a sequence block complementary to nucleotides 3-9 of bantam, including up to a single wobble $\mathrm{G}: \mathrm{U}$ pairing. These putative bantam binding sites would in principle be similar to those additional functional bantam sites in the hid 3' UTR characterized above. A list of 120 genes was compiled from the D. melanogaster genome that satisfied these criteria. Interestingly, TargetScan finds only two of these 120 candidate genes in the list of 74 candidate genes identified. The lack of congruence between these data sets likely results from the fact that genes identified by TargetScan must contain a canonical seed target sequence (with six or more contiguous nucleotides complementary to positions 1-8 of bantam) and be conserved in the 3' UTR of orthologous genes. Notably, our search algorithm did not include requirements for site conservation, for accessible structure in the target RNA or for pairing interactions with the $3^{\prime}$ end of the miRNA sequence. To ask whether these $3^{\prime}$ UTRs might respond to bantam within the cell, we chose three candidate $3^{\prime}$ UTRs [nct, I(3)73Ah, and CG34131] for further analysis, none of which contained a canonical seed match to nucleotides 1-8 of bantam. Strikingly, all three 3' UTRs conferred about twofold in regulation to the R-Luc reporter as assessed by the application of the anti-bantam oligonucleotide in the S2 cell culture system (Fig. 4B).

\section{DISCUSSION}

Here we describe the characterization of a system for the analysis of miRNA-mediated gene regulation in $D$. melanogaster S2 cells based on direct comparison of reporter constructs bearing both synthetic and natural 3' UTRs
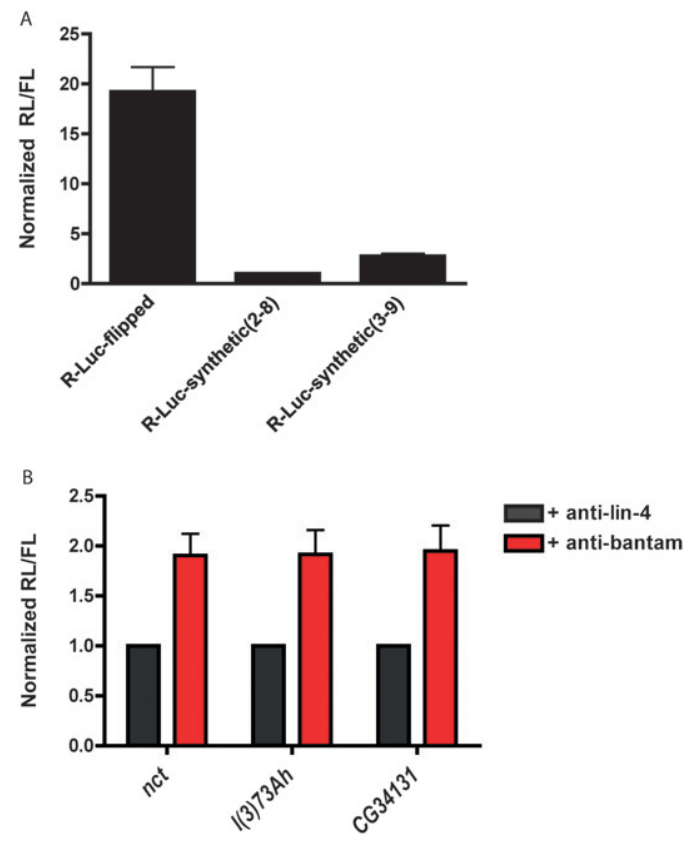

FIGURE 4. Regulation of constructs containing putative target sites that rely on complementary pairing with nucleotides 3-9 of bantam. (A) Constructs with synthetic 3' UTRs harboring binding sites with complementarity to either nucleotides $2-8$ or 3-9 of bantam are repressed to similar degrees. R-Luc-synthetic(2-8), R-Luc-synthetic(3-9), or R-Luc-flipped reporter plasmids were transfected (5 ng/well) along with F-Luc as the transfection control in D. melanogaster S2 cells. Both R-Luc-synthetic(2-8) and R-Luc-synthetic(39) constructs were repressed to similar extents relative to control RLuc-flipped. (B) Full-length 3' UTRs of three candidate genes, nct, $I(3) 73 A h$, and CG34131, were individually appended to Renilla luciferase and tested for bantam repression by co-transfecting with either anti-bantam oligonucleotide or control anti-lin-4. Normalized Renilla values in the presence of anti-bantam were divided by values in the presence of control anti-lin-4 (+anti-lin-4 = 1.0).

derived from the hid gene. In addition to developing critical tools for deciphering mechanism for this important form of gene regulation, our quantitative approaches have yielded a number of important insights. We show here that $2^{\prime}$-Omethyl antisense oligoribonucleotides (anti-miRNAs) can be used to effectively gauge the contribution of a given miRNA to regulation of a gene in a chosen system. As we first demonstrate for our synthetic reporter constructs (RLuc-synthetic and R-Luc-flipped), the application of antibantam oligonucleotides derepresses the R-Luc-synthetic construct to a similar degree (30- to 40-fold) as the removal of bantam complementary sequences from the $3^{\prime}$ UTR. We note that this level of miRNA-mediated regulation is high, but only is achieved when the key components are provided at relatively low concentrations (Fig. 1B,D). This sensitivity observed under conditions of low reporter mRNA input is likely to reflect limitations in the endogenous expression levels in S2 cells of bantam-specific miRNA machinery (Mathonnet et al. 2007). This interpretation is supported by the additional observation that overexpression of 
bantam under less sensitive conditions (high amounts of reporter) increases the extent of repression.

The equivalence in response of the synthetic reporter construct to target site removal and to anti-bantam oligonucleotide suggested that the application of antimiRNA could be used as a reliable measure of the contribution of a specific miRNA to repression in a given system. The initial nonequivalence of these data for the natural 3' UTR constructs (R-Luc-hid and R-Luc-hids4) was thus disconcerting. While the application of antibantam to the R-Luc-hid construct routinely derepressed expression by four- to sevenfold, the removal of the target sites in hid identified by a number of the routinely used algorithms (including TargetScan and Pictar/PITA) failed to yield a fully derepressed "control" construct. These inconsistencies ultimately led us to relax the stringency of seed definition to include matches to other seed registers of the miRNA (specifically registers $3-9$ and $4-10$ ) to ask how many other potential complementary target sequences might be identified. In the hid gene that we were studying, for example, this analysis identified two independent sites with matches to the 3-9 register. We were struck by the fact that the removal of these two 3-9 register seed match sites had a substantial effect on expression of the reporter construct, nicely derepressing luciferase expression to levels comparable to those observed in the presence of antibantam (Fig. 2B). While many laboratories have used the anti-miRNA approach to confirm the involvement of a given miRNA in a process (Leaman et al. 2005), we argue here for its utility as a more quantitative tool.

The system that we describe here provides us with a starting point for mechanistic dissection of miRNAmediated gene regulation. While earlier mechanistic studies have often depended on relatively small effects (as little as twofold), the larger effects that we observe (30- to 40-fold) should allow for more subtle features of regulation to be analyzed. More importantly, perhaps, our data suggest that miRNAs may contribute to the expression profile of even greater numbers of genes in vivo than previously anticipated. Current algorithms have focused on the canonical seed element (featuring complementarity with nucleotides at positions 1-8 of the miRNA) and on cross-species conservation. Our system provides evidence to suggest that the standard seed definition may be overly narrow. Indeed, each of the three genes that we chose to analyze in our reporter system to assess the potential function of a 3-9 register seed pairing responded to bantam in S2 cells (Fig. $4 \mathrm{~B})$. We note that some of the earliest bioinformatic analyses took an unbiased approach and considered the possibility of the full range of complementary registers contributing to miRNA-mediated regulation (Lewis et al. 2003). However, because the predicted signal:noise ratio for the 3-9 register targets was weaker than for the $1-7$ and 2-8 registers, consideration of these registers appears to have been abandoned at this early stage. Subsequent bioinfor- matic studies appear to adopt the seed definition originally proposed in Lewis et al. (2003) and refined in subsequent publications by this group (Lewis et al. 2005). Interestingly, however, miRNAs themselves seem to be conserved through position 9 (Lim et al. 2003), as well as their targets (Lewis et al. 2003), and microarray studies aimed at experimentally identifying miRNA target genes provided evidence that the 3-8 register is important for in vivo targeting (Lim et al. 2005). It is important to emphasize that prediction algorithms must draw arbitrary lines that allow for reasonable confidence in the targets identifiedand as a consequence, valid targets are missed. However, if we simply extrapolate our data on potential novel bantam targets to the larger miRNA database, we can expect a substantial increase in the number of genes subject to regulation through the miRNA pathway.

The most comprehensive analyses to date of the specific features of miRNA binding sites and their effectiveness in vivo are detailed in several key publications from the Hobert laboratory (Didiano and Hobert 2006, 2008). These studies have utilized GFP reporter genes fused to various $C$. elegans 3' UTRs to monitor endogenously expressed lys-6-mediated regulation in a paired set of neuronal cells. The asymmetrical expression of lys-6 miRNA in left and right neuronal cells provides a compelling set of controls to support use of this assay for analysis of 3' UTR function. We note, however, that because the GFP measurement in the worm is essentially a digital read-out, rather than a graded, analog one, the linear range of the assay may be somewhat limited.

These studies generally argued that the target identification programs are overly optimistic in predicting target genes with in vivo relevance. This point was most clearly demonstrated by the observation that none of 13 putative lys- 6 target $3^{\prime}$ UTRs tested, as identified by a number of prediction algorithms, exhibited repression by lys-6 in their in vivo assay (Didiano and Hobert 2006). While, at first glance, the conclusions of our studies seem at odds with those from the Hobert laboratory, these differences provide insight into the complexities of posttranscriptional gene regulation. We would suggest that the studies in C. elegans highlight the complexities of miRNAmediated gene regulation in an authentic, but rather specialized in vivo context. Multi-layered, combinatorial control of post-transcriptional gene regulation is anticipated based on the extensive length of $3^{\prime}$ UTRs in eukaryotic cells and the complex developmental patterns of gene expression that are observed. Our studies, performed in a cell culture system, but one that still depends on endogenous miRNA and the related machinery, likely highlight some of the simplest features of this regulation. In our sensitive reporter assay, a single miRNA binding site appears to contribute on the order of twofold to gene regulation, consistent with reports from other systems (Doench et al. 2003; Pillai et al. 2005). Our studies further argue that target prediction programs may miss an entire class of potential targets (the 3-9 register seed match) because of the difficulties in distinguishing signal from noise for these cases. 
Indeed, recent proteomic approaches argue that nonconserved target sequences may also respond to miRNAs in vivo, thus providing support for more extensive regulation by miRNAs than currently predicted (Baek et al. 2008; Selbach et al. 2008). Empirical approaches for target identification for this ubiquitous class of regulators, the miRNAs, likely still have much to offer at this relatively early stage of the prediction game.

\section{MATERIALS AND METHODS}

\section{DNA constructs}

Reporter constructs were generated by amplifying the 3' UTR of indicated $D$. melanogaster genes from genomic DNA followed by cloning of the PCR product downstream from the Renilla luciferase coding region using $\mathrm{KpnI}$ and BamHI restriction sites (Promega actin pRL vector). To generate D. melanogaster 3' UTR reporter constructs carrying seed element deletions, six to seven nucleotides complementary to the miRNA seed sequence were removed from the indicated 3' UTR using QuikChange II XL sitedirected mutagenesis kit (Stratagene). Reporters with synthetic 3' UTRs were generated by ligating overlapping oligos into KpnI and NotI restriction sites downstream from the luciferase coding region (Promega actin pRL vector except in the cases of R-Lucsynthetic(2-8), R-Luc-synthetic(3-9) which utilized a Promega copia pRL vector). PCR primers employed were AGCGCAGGAG ACGTGTAATCG and GTGATCAGCTTGCCATAATCC (hid), GTAACAGCTCTCTAAGCGCAGG and GCTGCTCAAGCGTGA CATTGTC ( Gli), GTGAGCTGATCCCGCCGTTAAAG and GGA TCATCGTGTCAGTGGAG $(s c a)$, AGGGCATTCCATATATACGC and CTCGCTGTGGACACACTTTC $(H L H m \delta)$, ATTTCTAGTTTTA CACCGCAAAAC and CACTGCACTCATACGAGTACCG (TER94), TGTCACAACTGCCACAGCG and CTTCAATAACGTGGCAGCCG $(n c t)$, CCACTGTTTTAGTATAATGCC and GGATCGGTTGAGA AGATTGC [I(3)73Ah], AAACTATGCTGAAGTCAACG and CCG TGAGCCCACATTCCG (CG34131).

\section{Cell culture}

S2 cells were cultured in Drosophila SFM medium (Invitrogen) supplemented with 100 units/mL of penicillin and streptomycin (Cambrex Bioscience) and $16 \mathrm{mM}$ L-Glutamine (Invitrogen). S2R+ cells used in RNAi experiments were cultured in Schneider's Drosophila medium (Invitrogen) supplemented with 10\% heat-inactivated FBS (Invitrogen) and 100 units/mL penicillin and streptomycin.

\section{Transfection}

S2 cells were plated at a density of $\sim 5 \times 10^{5}$ cells per well in 24 well plates and transfected using Effectene reagent (Qiagen). Indicated amounts of R-Luc reporter constructs were transfected along with $1 \mathrm{ng}$ of F-Luc control. Luciferase activities were measured $48 \mathrm{~h}$ following transfection using dual-luciferase reporter assay system (Promega).

\section{RNA interference, bathing of S2R+ cells, and mRNA quantification}

$\mathrm{S} 2 \mathrm{R}+$ cells were seeded at a density of $1-3 \times 10^{5}$ cells/well on day prior to use. Cells were treated with $15 \mu \mathrm{g} / \mathrm{mL}$ dsRNAs encompassing $\sim 500 \mathrm{nt}$ of the $A G O 1$ and $A G O 2$ coding regions or control YFP on days 0 and 3 in serum-free media for $1 \mathrm{~h}$ followed by addition of an equal volume of media containing 20\% FBS. Cells were transfected with R-Luc-synthetic or R-Luc-flipped reporter constructs along with F-Luc control on day 6, and luciferase activities were measured on day 8. AGO1 and AGO2 mRNA levels were quantified by qRT-PCR using the iQ5 iCycler system (Bio-Rad) and iQ SYBR Green Supermix (Bio-Rad).

\section{SUPPLEMENTAL MATERIAL}

Supplemental material can be found at http://www.rnajournal.org.

\section{ACKNOWLEDGMENTS}

We thank Michael Beer for computational assistance in identifying bantam complementary regions within annotated $D$. melanogaster 3' UTRs, Junho Hur and Sofia Vidal-Cardenas for help in the construction of initial deletion constructs, and Luisa Cochella and Joshua Mendell for helpful comments on the manuscript. This work was supported by a Life Sciences Research Foundation postdoctoral fellowship (A.N.).

Received January 9, 2009; accepted January 22, 2009.

\section{REFERENCES}

Aravin, A.A., Lagos-Quintana, M., Yalcin, A., Zavolan, M., Marks, D., Snyder, B., Gaasterland, T., Meyer, J., and Tuschl, T. 2003. The small RNA profile during Drosophila melanogaster development. Dev. Cell 5: 337-350.

Baek, D., Villen, J., Shin, C., Camargo, F.D., Gygi, S.P., and Bartel, D.P. 2008. The impact of microRNAs on protein output. Nature 455: 64-71.

Bagga, S., Bracht, J., Hunter, S., Massirer, K., Holtz, J., Eachus, R., and Pasquinelli, A.E. 2005. Regulation by let-7 and lin-4 miRNAs results in target mRNA degradation. Cell 122: 553-563.

Behm-Ansmant, I., Rehwinkel, J., Doerks, T., Stark, A., Bork, P., and Izaurralde, E. 2006a. mRNA degradation by miRNAs and GW182 requires both CCR4:NOT deadenylase and DCP1:DCP2 decapping complexes. Genes \& Dev. 20: 1885-1898.

Behm-Ansmant, I., Rehwinkel, J., and Izaurralde, E. 2006b. MicroRNAs silence gene expression by repressing protein expression and/or by promoting mRNA decay. Cold Spring Harb. Symp. Quant. Biol. 71: 523-530.

Brennecke, J., Hipfner, D.R., Stark, A., Russell, R.B., and Cohen, S.M. 2003. bantam encodes a developmentally regulated microRNA that controls cell proliferation and regulates the proapoptotic gene hid in Drosophila. Cell 113: 25-36.

Brennecke, J., Stark, A., Russell, R.B., and Cohen, S.M. 2005. Principles of microRNA-target recognition. PLoS Biol. 3: e85. doi: 10.1371/journal.pbio.0030085.

Bushati, N. and Cohen, S.M. 2007. MicroRNA functions. Annu. Rev. Cell Dev. Biol. 23: 175-205.

Chendrimada, T.P., Finn, K.J., Ji, X., Baillat, D., Gregory, R.I., Liebhaber, S.A., Pasquinelli, A.E., and Shiekhattar, R. 2007. MicroRNA silencing through RISC recruitment of eIF6. Nature 447: 823-828.

Didiano, D. and Hobert, O. 2006. Perfect seed pairing is not a generally reliable predictor for miRNA-target interactions. Nat. Struct. Mol. Biol. 13: 849-851.

Didiano, D. and Hobert, O. 2008. Molecular architecture of a miRNA-regulated 3' UTR. RNA 14: 1297-1317. 
Doench, J.G. and Sharp, P.A. 2004. Specificity of microRNA target selection in translational repression. Genes \& Dev. 18: 504-511.

Doench, J.G., Petersen, C.P., and Sharp, P.A. 2003. siRNAs can function as miRNAs. Genes \& Dev. 17: 438-442.

Esquela-Kerscher, A. and Slack, F.J. 2006. Oncomirs-MicroRNAs with a role in cancer. Nat. Rev. Cancer 6: 259-269.

Eulalio, A., Huntzinger, E., and Izaurralde, E. 2008. GW182 interaction with Argonaute is essential for miRNA-mediated translational repression and mRNA decay. Nat. Struct. Mol. Biol. 15: 346-353.

Farh, K.K., Grimson, A., Jan, C., Lewis, B.P., Johnston, W.K. Lim, L.P., Burge, C.B., and Bartel, D.P. 2005. The widespread impact of mammalian microRNAs on mRNA repression and evolution. Science 310: 1817-1821.

Giraldez, A.J., Mishima, Y., Rihel, J., Grocock, R.J., Van Dongen, S., Inoue, K., Enright, A.J., and Schier, A.F. 2006. Zebrafish MiR-430 promotes deadenylation and clearance of maternal mRNAs. Science 312: 75-79.

Grether, M.E., Abrams, J.M., Agapite, J., White, K., and Steller, H. 1995. The head involution defective gene of Drosophila melanogaster functions in programmed cell death. Genes \& Dev. 9: 1694-1708.

Griffiths-Jones, S., Grocock, R.J., van Dongen, S., Bateman, A., and Enright, A.J. 2006. miRBase: MicroRNA sequences, targets and gene nomenclature. Nucleic Acids Res. 34: D140-D144.

Grün, D., Wang, Y.L., Langenberger, D., Gunsalus, K.C., and Rajewsky, N. 2005. MicroRNA target predictions across seven Drosophila species and comparison to mammalian targets. PLoS Comput. Biol. 1: e13. doi: 10.1371/journal.pcbi.0010013.

Jackson, R.J. and Standart, N. 2007. How do microRNAs regulate gene expression? Sci. STKE 2007: re1. doi: 10.1126/stke.3672007re1.

Kato, M. and Slack, F.J. 2008. MicroRNAs: Small molecules with big roles-C. elegans to human cancer. Biol. Cell 100: 71-81.

Kertesz, M., Iovino, N., Unnerstall, U., Gaul, U., and Segal, E. 2007. The role of site accessibility in microRNA target recognition. Nat. Genet. 39: 1278-1284.

Kiriakidou, M., Tan, G.S., Lamprinaki, S., De Planell-Saguer, M., Nelson, P.T., and Mourelatos, Z. 2007. An mRNA m7G cap binding-like motif within human Ago2 represses translation. Cell 129: 1141-1151.

Krek, A., Grün, D., Poy, M.N., Wolf, R., Rosenberg, L., Epstein, E.J., MacMenamin, P., da Piedade, I., Gunsalus, K.C., Stoffel, M., et al. 2005. Combinatorial microRNA target predictions. Nat. Genet. 37: 495-500.

Krutzfeldt, J., Rajewsky, N., Braich, R., Rajeev, K.G., Tuschl, T., Manoharan, M., and Stoffel, M. 2005. Silencing of microRNAs in vivo with "antagomirs." Nature 438: 685-689.

Leaman, D., Chen, P.Y., Fak, J., Yalcin, A., Pearce, M., Unnerstall, U., Marks, D.S., Sander, C., Tuschl, T., and Gaul, U. 2005. Antisensemediated depletion reveals essential and specific functions of microRNAs in Drosophila development. Cell 121: 1097-1108.

Lee, R.C., Feinbaum, R.L., and Ambros, V. 1993. The C. elegans heterochronic gene lin-4 encodes small RNAs with antisense complementarity to lin-14. Cell 75: 843-854.

Lewis, B.P., Shih, I.H., Jones-Rhoades, M.W., Bartel, D.P., and Burge, C.B. 2003. Prediction of mammalian microRNA targets. Cell 115: 787-798.

Lewis, B.P., Burge, C.B., and Bartel, D.P. 2005. Conserved seed pairing, often flanked by adenosines, indicates that thousands of human genes are microRNA targets. Cell 120: 15-20.

Lim, L.P., Lau, N.C., Weinstein, E.G., Abdelhakim, A., Yekta, S., Rhoades, M.W., Burge, C.B., and Bartel, D.P. 2003. The microRNAs of Caenorhabditis elegans. Genes \& Dev. 17: 991-1008.

Lim, L.P., Lau, N.C., Garrett-Engele, P., Grimson, A., Schelter, J.M., Castle, J., Bartel, D.P., Linsley, P.S., and Johnson, J.M. 2005. Microarray analysis shows that some microRNAs downregulate large numbers of target mRNAs. Nature 433: 769-773.
Mathonnet, G., Fabian, M.R., Svitkin, Y.V., Parsyan, A., Huck, L., Murata, T., Biffo, S., Merrick, W.C., Darzynkiewicz, E., Pillai, R.S., et al. 2007. MicroRNA inhibition of translation initiation in vitro by targeting the cap-binding complex eIF4F. Science 317: 1764-1767.

Meister, G., Landthaler, M., Dorsett, Y., and Tuschl, T. 2004. Sequence-specific inhibition of microRNA- and siRNA-induced RNA silencing. RNA 10: 544-550.

Nilsen, T.W. 2007. Mechanisms of microRNA-mediated gene regulation in animal cells. Trends Genet. 23: 243-249.

Nissan, T. and Parker, R. 2008. Computational analysis of miRNAmediated repression of translation: Implications for models of translation initiation inhibition. RNA 14: 1480-1491.

Nottrott, S., Simard, M.J., and Richter, J.D. 2006. Human let-7a miRNA blocks protein production on actively translating polyribosomes. Nat. Struct. Mol. Biol. 13: 1108-1114.

Olsen, P.H. and Ambros, V. 1999. The lin-4 regulatory RNA controls developmental timing in Caenorhabditis elegans by blocking LIN-14 protein synthesis after the initiation of translation. Dev. Biol. 216: 671-680.

Petersen, C.P., Bordeleau, M.E., Pelletier, J., and Sharp, P.A. 2006. Short RNAs repress translation after initiation in mammalian cells. Mol. Cell 21: 533-542.

Pillai, R.S., Bhattacharyya, S.N., Artus, C.G., Zoller, T., Cougot, N., Basyuk, E., Bertrand, E., and Filipowicz, W. 2005. Inhibition of translational initiation by Let-7 MicroRNA in human cells. Science 309: 1573-1576.

Pillai, R.S., Bhattacharyya, S.N., and Filipowicz, W. 2007. Repression of protein synthesis by miRNAs: How many mechanisms? Trends Cell Biol. 17: 118-126.

Rehwinkel, J., Natalin, P., Stark, A., Brennecke, J., Cohen, S.M., and Izaurralde, E. 2006. Genome-wide analysis of mRNAs regulated by Drosha and Argonaute proteins in Drosophila melanogaster. Mol. Cell. Biol. 26: 2965-2975.

Reinhart, B.J., Slack, F.J., Basson, M., Pasquinelli, A.E., Bettinger, J.C., Rougvie, A.E., Horvitz, H.R., and Ruvkun, G. 2000. The 21nucleotide let-7 RNA regulates developmental timing in Caenorhabditis elegans. Nature 403: 901-906.

Selbach, M., Schwanhäusser, B., Thierfelder, N., Fang, Z., Khanin, R., and Rajewsky, N. 2008. Widespread changes in protein synthesis induced by microRNAs. Nature 455: 58-63.

Stark, A., Brennecke, J., Russell, R.B., and Cohen, S.M. 2003. Identification of Drosophila microRNA targets. PLoS Biol. 1: e60. doi: 10.1371/journal.pbio.0000060.

Stark, A., Brennecke, J., Bushati, N., Russell, R.B., and Cohen, S.M. 2005. Animal microRNAs confer robustness to gene expression and have a significant impact on $3^{\prime}$ UTR evolution. Cell 123: $1133-1146$.

Thermann, R. and Hentze, M.W. 2007. Drosophila miR2 induces pseudo-polysomes and inhibits translation initiation. Nature 447: 875-878.

Vasudevan, S., Tong, Y., and Steitz, J.A. 2007. Switching from repression to activation: MicroRNAs can up-regulate translation. Science 318: 1931-1934.

Wang, B., Love, T.M., Call, M.E., Doench, J.G., and Novina, C.D. 2006. Recapitulation of short RNA-directed translational gene silencing in vitro. Mol. Cell 22: 553-560.

Wightman, B., Ha, I., and Ruvkun, G. 1993. Posttranscriptional regulation of the heterochronic gene lin-14 by lin-4 mediates temporal pattern formation in C. elegans. Cell 75: 855-862.

Wu, L., Fan, J., and Belasco, J.G. 2006. MicroRNAs direct rapid deadenylation of mRNA. Proc. Natl. Acad. Sci. 103: 40344039.

Zeng, Y., Wagner, E.J., and Cullen, B.R. 2002. Both natural and designed micro RNAs can inhibit the expression of cognate mRNAs when expressed in human cells. Mol. Cell 9: 13271333. 

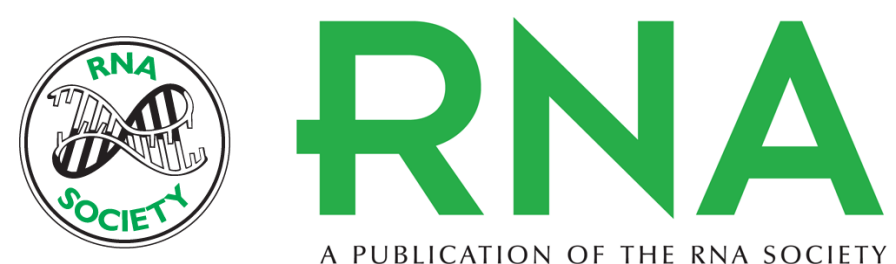

A PUBLICATION OF THE RNA SOCIETY

\section{An expanded seed sequence definition accounts for full regulation of the hid 3' UTR by bantam miRNA}

Ali Nahvi, Christopher J. Shoemaker and Rachel Green

RNA 2009 15: 814-822 originally published online March 12, 2009

Access the most recent version at doi:10.1261/rna.1565109

\section{Supplemental http://rnajournal.cshlp.org/content/suppl/2009/03/16/rna.1565109.DC1 \\ Material}

References This article cites 52 articles, 17 of which can be accessed free at:

http://rnajournal.cshlp.org/content/15/5/814.full.html\#ref-list-1

\section{License}

Email Alerting Receive free email alerts when new articles cite this article - sign up in the box at the Service top right corner of the article or click here.

To subscribe to RNA go to:

http://rnajournal.cshlp.org/subscriptions 